\title{
Discordance of the PAM50 Intrinsic Subtypes Compared with Immunohistochemistry-Based Surrogate in Breast Cancer Patients: Potential Implication of Genomic Alterations of Discordance
}

\author{
Hee Kyung Kim, MD ${ }^{1,2}$ \\ Kyung Hee Park, $\mathrm{MS}^{3}$ \\ Youjin Kim, MD' \\ Song Ee Park, MD \\ Han Sang Lee, MD ${ }^{1}$ \\ Sung Won Lim, MD \\ Jang Ho Cho, MD' \\ Ji-Yeon Kim, MD ${ }^{1}$ \\ Jeong Eon Lee, MD, PhD ${ }^{4}$ \\ Jin Seok Ahn, MD, PhD' \\ Young-Hyuck Im, MD, PhD 1 \\ Jong Han Yu, MD, PhD ${ }^{4}$ \\ Yeon Hee Park, MD, PhD ${ }^{1}$
}

*A list author's affiliations appears at the end of the paper.

\section{Purpose}

We aimed to analyze the discordance between immunohistochemistry (IHC)-based surrogate subtyping and PAM50 intrinsic subtypes and to assess overall survival (OS) according to discordance.

\section{Materials and Methods}

A total of 607 patients were analyzed. Hormone receptor (HR) expression was evaluated by IHC, and human epidermal growth factor receptor 2 (HER2) expression was analyzed by $\mathrm{IHC}$ and/or fluorescence in situ hybridization. PAM50 intrinsic subtypes were determined according to 50 cancer genes using the NanoString $n$ Counter Analysis System. We matched concordant tumor as luminal A and HR+/HER2-, luminal B and HR+/HER2+, HR-/HER2+ and HER2-enriched, and triple-negative breast cancer (TNBC) and normal- or basal-like. We used Ion Ampliseq Cancer Panel v2 was used to identify the genomic alteration related with discordance. The Kaplan-Meier method was used to estimate OS.

\section{Results}

In total, 233 patients (38.4\%) were discordant between IHC-based subtype and PAM50 intrinsic subtype. Using targeted sequencing, we detected somatic mutation-related discordant breast cancer including the $\mathrm{VHL}$ gene in the HR+/HER2- group (31\% in concordant group, $0 \%$ in discordant group, $\mathrm{p}=0.03$ ) and the IDH and $R E T$ genes ( $7 \%$ vs. $12 \%, p=0.02$ and $0 \%$ vs. $25 \%, p=0.02$, respectively) in the TNBC group. Among the luminal A/B patients with a discordant result had significantly worse OS (median OS, 73.6 months vs. not reached; $p<0.001$ ), and among the patients with HR positivity, the basal-like group as determined by PAM50 showed significantly inferior OS compared to other intrinsic subtypes (5-year 0 rate, $92.2 \%$ vs. $75.6 \%$; $p=0.01$ ).

\section{Conclusion}

A substantial portion of patients showed discrepancy between IHC subtype and PAM50 intrinsic subtype in our study. The survival analysis demonstrated that current IHC-based classification could mislead the treatment and result in poor outcome. Current guidelines for IHC might be updated accordingly.

\section{Key words}

Breast neoplasms, PAM50, Immunohistochemistry 


\section{Introduction}

Despite advances in cancer treatment, breast cancer is the leading cause of cancer death in women [1]. In recent decades, intrinsic molecular subtypes have been established to understand the biological complexity of breast cancer. The intrinsic subtypes of breast cancer are defined into five groups of luminal A, luminal B, human epidermal growth factor receptor 2 (HER2)-enriched, basal-like, and normal-like [2-4]. Gene-expression profiling and tissue microarray have defined the intrinsic subtypes, and several studies with varying numbers of genes have been performed to develop a genomic test $[5,6]$. Prior studies have shown significant improvement in terms of risk factors, incidence, prognosis, and responses to systemic therapies $[7,8]$.

Among the prior genomic tests, Parker et al. [7] reported a PAM50 assay using GEP, which is widely used in a clinical setting $[9,10]$. The PAM50 assay can be used to assess the expression levels of 50 genes in surgically resected breast cancer tissue including formalin-fixed paraffin-embedded (FFPE) tissue in hormone receptor (HR) positive patients [4,11]. The PAM50 assay (Prosigna Breast Cancer Gene Signature Assay, NanoString Technologies) was approved by the U.S. Food and Drug Administration (FDA) in September 2013. However, there is currently no practical molecular assay for intrinsic subtypes in real-world practice that addresses the problems of cost and run-time. In routine pathological works, breast cancer subtypes are classified by immunohistochemistry (IHC) to design treatments, predict prognosis of patients, and enroll in clinical trials.

Breast cancer patients in Asian populations, including Korea, have different demographic features compared to Caucasian patients. The majority of breast cancer patients are premenopausal, and the peak incidence age is in the $40 \mathrm{~s}$ $[12,13]$. Also, premenopausal breast cancer patients are known to have poor prognosis and aggressive clinical behavior [14]. However, most of the approved commercial assays for intrinsic subtypes have not been evaluated in an Asian population [15]. The discordance of IHC-based subtype with intrinsic subtype has not been investigated in Asian populations, and survival considering discordance has not been evaluated. Hence, we aimed to assess the discordance between IHC-based subtype and PAM50 intrinsic subtype and to evaluate overall survival (OS) according to discordance. We additionally aimed to investigate the genetic mutations that are associated with discordance in Korean breast cancer patients.

\section{Materials and Methods}

\section{Patients}

We collected data from the electronic medical records and all tumor samples from patients who underwent curative surgery or tumor biopsy at Samsung Medical Center $(n=605)$. The patients were enrolled in five retrospective studies and one clinical trial. The information from the six clinical studies is shown in S1 Table. Data comprised sex, age, TNM staging, HR status (expression of estrogen receptor and progesterone receptor), HER2 expression (confirmed by fluorescence in situ hybridization or silver in situ hybridization), menopausal status, date of diagnosis (curative surgery or first tumor biopsy), date of death, and date of last follow-up.

\section{DNA/RNA extraction}

A total of 605 tissue samples with a tumor cell percentage greater than $75 \%$ (from 4-mm unstained sections) were dissected under a microscope based on comparison to a hematoxylin and eosin-stained slide. Genomic DNA was extracted using the Qiagen DNA FFPE Tissue Kit (Qiagen, Hilden, Germany), and total RNA was extracted using the High Pure RNA Paraffin kit (Roche Diagnostics, Mannheim, Germany), according to the manufacturer's instructions. After extraction, we measured DNA and RNA concentration using a spectrophotometer (ND1000, NanoDrop Technologies, Thermo Fisher Scientific, Waltham, MA). Each sample was then quantified using a Qubit fluorometer (Life Technologies, Carlsbad, CA). Samples with less than $10 \mathrm{ng} / \mu \mathrm{L}$ of genomic DNA and less than $50 \mathrm{ng} / \mu \mathrm{L}$ of total RNA, even after concentration using a SpeedVac concentrator (Thermo Scientific, Waltham, MA) were excluded from downstream analysis.

\section{Next-generation sequencing using Ion Ampliseq Cancer Panel v2}

Using the Ion Torrent Personal Genome Machine (Ion PGM, Life Technologies, Carlsbad, CA) Cancer Panel v2 (S2 Table) after DNA isolation from FFPE samples, we sequenced 2,855 loci from 50 cancer-related genes to identify genetic mutations in 118 samples from breast cancer patients. Libraries were constructed using the Ion AmpliSeq Panels pool (Life Technologies) with a 10-ng DNA sample per pool. The amplicons were then ligated to Ion Xpress Barcode Adapters and purified. Next, multiplexed barcoded libraries were enriched by clonal amplification using emulsion polymerase chain reaction on Ion Sphere particles (Ion PGM Template OT2 $200 \mathrm{Kit}$, Life Technologies) and loaded onto an Ion 316 Chip. Massively parallel sequencing was carried out on 
the Ion PGM using the Ion PGM Sequencing $200 \mathrm{Kit}$ v2. The Ion AmpliSeq Cancer Hotspot Panel v2 (http:// www.lifetechnologies.com) covered hot-spot regions of 50 oncogenes and tumor suppressor genes. The primary filtering process was carried out using the Torrent Suite v3.6.0 and the Ion Torrent Variant Caller v3.6 software. The pipeline included signaling processing, base calling, quality score assignment, adapter trimming, read alignment to human genome 19 references, mapping $\mathrm{QC}$, coverage analysis, and variant calling. For variant detection, a minimum coverage of 100 reads must be achieved, and at least $5 \%$ of mutant reads were selected as variants. Variant calls were further analyzed using the ANNOVAR, which included variant filtering and annotation using the COSMIC database, dbSNP build 137, and amino acid change information.

\section{PAM50 intrinsic subtype prediction}

PAM50 gene expression was measured on the NanoString nCounter Analysis System (NanoString Technologies, Seattle, WA). The system measures the relative abundance of each mRNA transcript of interest using a multiplexed hybridization assay, and digital readouts of fluorescent barcoded probes that are hybridized to each transcript are created [16]. Intrinsic subtype classification was performed using the PAM50 predictor and was applied to the nearest PAM50 centroid algorithm Bioclassifier to predict the PAM50 subtypes, as described in Parker et al. [7]. To obtain more consistent results, we merged microarray expression data of The Cancer Genome Atlas (TCGA) breast cancers with our NanoString data after adjusting for batch effects using the ComBat algorithm [17] and applied the nearest PAM50 centroid algorithm Bioclassifier to predict PAM50 subtypes [7]. For all statistical tests, PAM50 subtype prediction was conducted using R ver. 3.5.1 (http://www.R-project.org/).

\section{Statistical analysis}

For analysis of the difference according to discordance, SPSS statistical software ver. 23 (IBM Corp., Armonk, NY) was used. All comparisons were examined by Fisher exact test. False discovery rate was applied for multiple testing correction. OS was measured from the date of curative surgery or first tumor biopsy to the date of death or last follow-up. The Kaplan-Meier method was used to estimate OS, and survival curves were compared by the log-rank test. A Cox proportional hazard regression model was used to assess the impact of the prognostic variable on OS. All tests were twotailed, and p-values $<0.05$ were considered significant.
Table 1. Patient characteristics

\begin{tabular}{lr} 
Characteristic & No. $(\%)(\mathbf{n}=607)$ \\
Age, median (range, yr) & $47(21-78)$ \\
Overall survival, median (range, yr) & $18.3(0-20.3)$ \\
Menopausal status (n=605) & \\
Premenopausal & $432(71.2)$ \\
$\quad$ Postmenopausal & $173(28.5)$ \\
Stage (n=605) & \\
I & $132(21.7)$ \\
II & $307(50.6)$ \\
III & $150(24.7)$ \\
IV & $16(2.6)$ \\
IHC & \\
HR+/HER2- & $279(46.0)$ \\
HR+/HER2+ & $65(10.7)$ \\
\hline HR-/HER2+ & $113(18.6)$ \\
TNBC & $150(24.7)$ \\
PAM50 & $181(29.8)$ \\
Luminal A & $102(16.8)$ \\
\hline Luminal B & $144(23.7)$ \\
\hline HER2-enriched & $149(24.5)$ \\
\hline Basal-like & $31(5.1)$ \\
\hline Normal-like &
\end{tabular}

IHC, immunohistochemistry; HR, hormone receptor; HER2, human epidermal growth factor receptor 2; TNBC, triplenegative breast cancer.

\section{Ethical statement}

This study was approved by the institutional review board of Samsung Medical Center, Seoul, Korea (IRB No. 2018-02004) with an informed consent waiver due to the use of archival tissues with retrospective clinical data.

\section{Results}

\section{Patient characteristics}

The patient characteristics are demonstrated in Table 1, and a total of 607 patients with breast cancer were analyzed. The median age of all patients was 47 years (range, 21 to 78 years), and approximately $70 \%$ of patients were premenopausal. Most patients had early breast cancer, and stage IV metastatic breast cancer was found in $2.6 \%$ of patients (16 of $605)$. The types of sample were $53 \%(321 / 605)$ fresh frozen tissue and 47\% (286/605) FFPE. The proportion of patients 

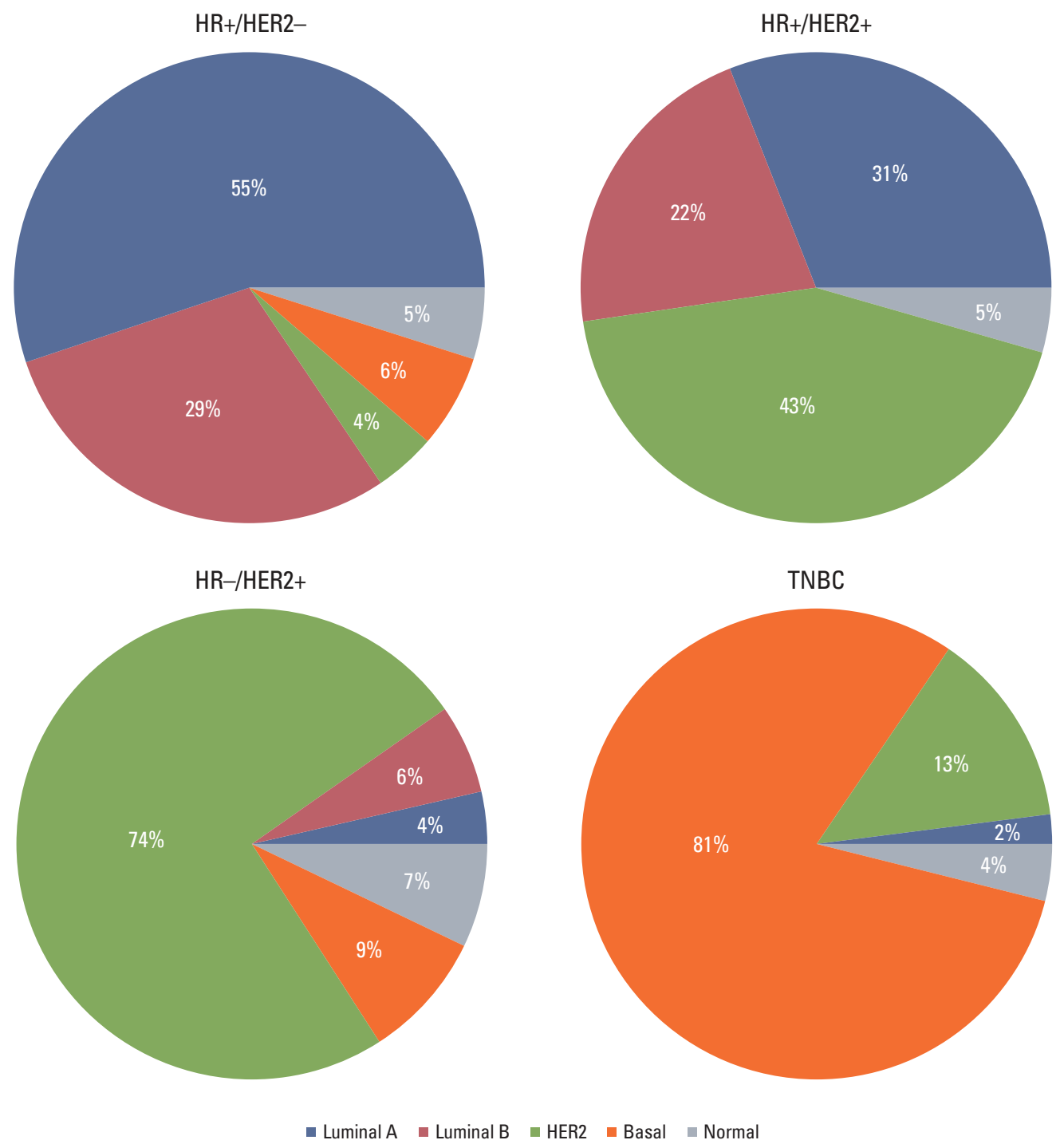

Fig. 1. Distribution of the PAM50 intrinsic subtypes within each immunohistochemistry-based group. HR, hormone receptor; HER2, human epidermal growth factor receptor 2; TNBC, triple-negative breast cancer.

within each study is depicted in S3 Fig. The proportions of HR+ / HER2-, HR+ / HER2+, HR-/HER2+, and triple-negative breast cancer (TNBC) in the study population were $46.0 \% .10 .7 \%, 18.6 \%$, and $24.7 \%$, respectively. According to PAM50 intrinsic subtypes, $46.6 \%$ were luminal A/B, $23.7 \%$ were HER2-enriched, $24.5 \%$ were basal-like, and $5.1 \%$ were normal-like type (Table 1).

\section{Pattern of discordance between IHC subtype and PAM50 intrinsic subtype was distinct among the IHC-based group}

In total, 233 patients $(38.4 \%)$ were discordant between IHC-based subtype and PAM50 intrinsic subtype. The dis- tribution of PAM50 intrinsic subtypes within each IHC subtype is depicted in Fig. 1. Among the 278 patients with $\mathrm{HR}+$ / HER2-, non-luminal A/B type was found in $4 \%$ with HER2enriched, $6 \%$ with basal-like, and $6 \%$ with normal-like type, respectively. Twenty-six percent of HR-/HER2+ patients were not HER2-enriched type. Of 151 TNBC patients, 2\% were luminal A type, and 13\% were HER2-enriched type. The majority of discordant patients were HR+ (176 of 234, $75.2 \%$ ), and $12.4 \%$ (29 of 234) were HER2+. Among the 23 TNBC patients with discordance, $86.7 \%$ (20 of 23 ) were classified as HER2-enriched type. 


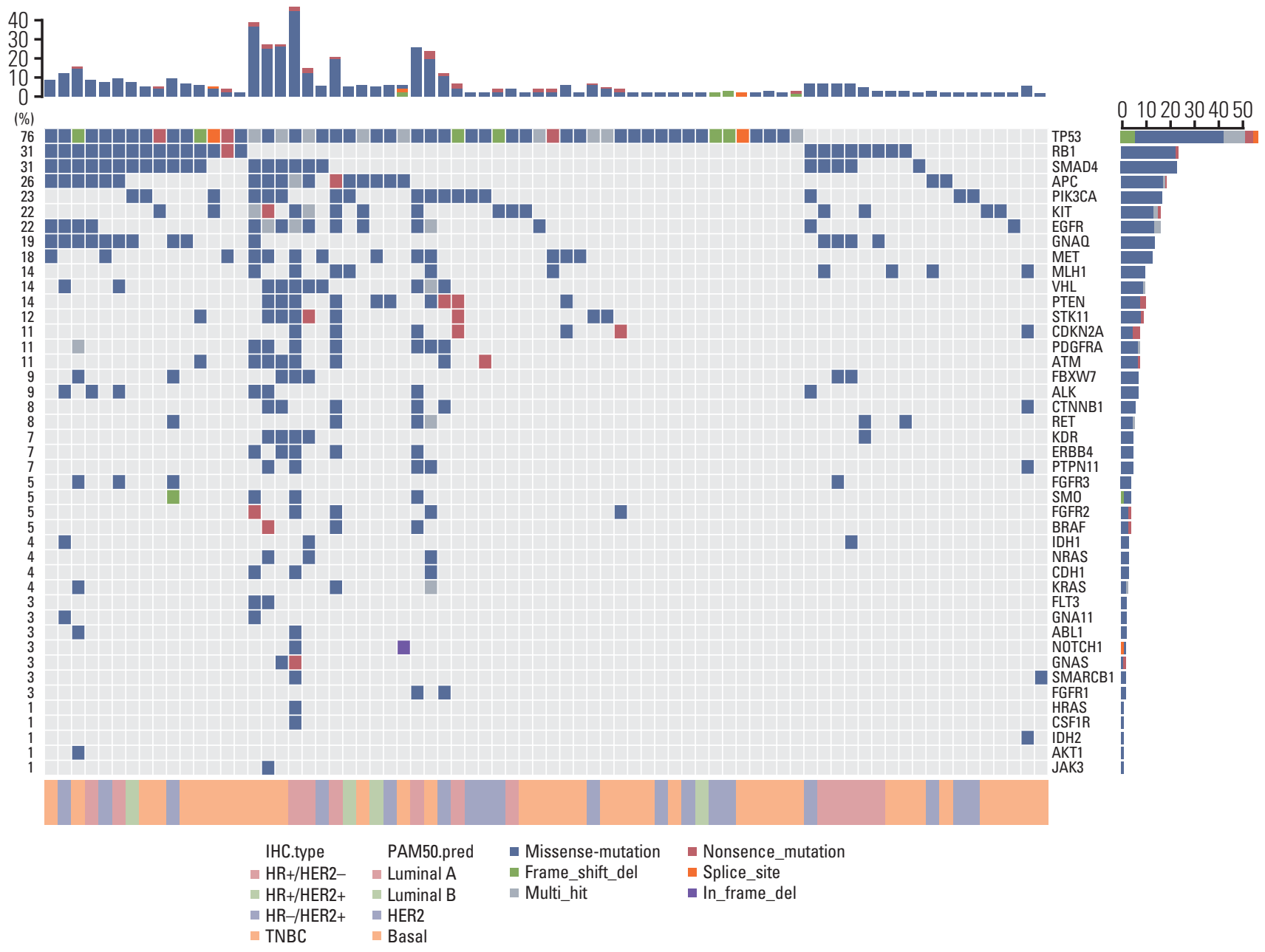

Fig. 2. Mutation pattern according to the frequency of 50 cancer-related genes: mutation map of concordant group (A) and mutation map of discordant group (B). HR, hormone receptor; HER2, human epidermal growth factor receptor 2; TNBC, triple-negative breast cancer. (Continued to the next page)

\section{DNA sequencing of concordant/discordant breast cancer shows heterogeneous mutation patterns}

To identify the patterns of gene expression associated with discordancy, we performed next-generation sequencing using the Ion Ampliseq cancer panel (minor allele frequency $>0.01$ ). Somatic single nucleotide variants and small insertion-deletions (indels) were most frequently observed as the types of genetic alterations (S4 Fig.). TP53 was the most common mutation $(67 \%)$, and the majority were missense mutations. Other frequent mutations in our cohort were $R B 1$ (28.8\%), SMAD4 (28\%), APC (25.4\%), and PIK3CA (21.2\%). Fig. 2 demonstrates the frequency of mutations in 50 genes among 118 patients. The frequency of mutations was not significantly different between the concordant and discordant groups $(\mathrm{p}=0.26)$.

\section{Significant difference in mutation patterns detected according to discordance}

Next, we compared the mutation profiles of concordant tumors $(n=83)$ and discordant tumors $(n=35)$ in order to identify gene expression signatures associated with discordance using the 50-cancer gene panel. Among HR+/HER2- patients, mutation in the VHL gene was significantly higher in concordant patients (31\% in concordant group, $0 \%$ in discordant group, $\mathrm{p}=0.03$ ) (Fig. 3A). Among TNBC patients, the IDH1 and RET genes were more frequently observed in the discordant group ( $7 \%$ vs. $12 \%, \mathrm{p}=0.02 ; 0 \%$ vs. $25 \%$, respectively; $\mathrm{p}=0.02$ ) (Fig. 3D). There was no significant difference 


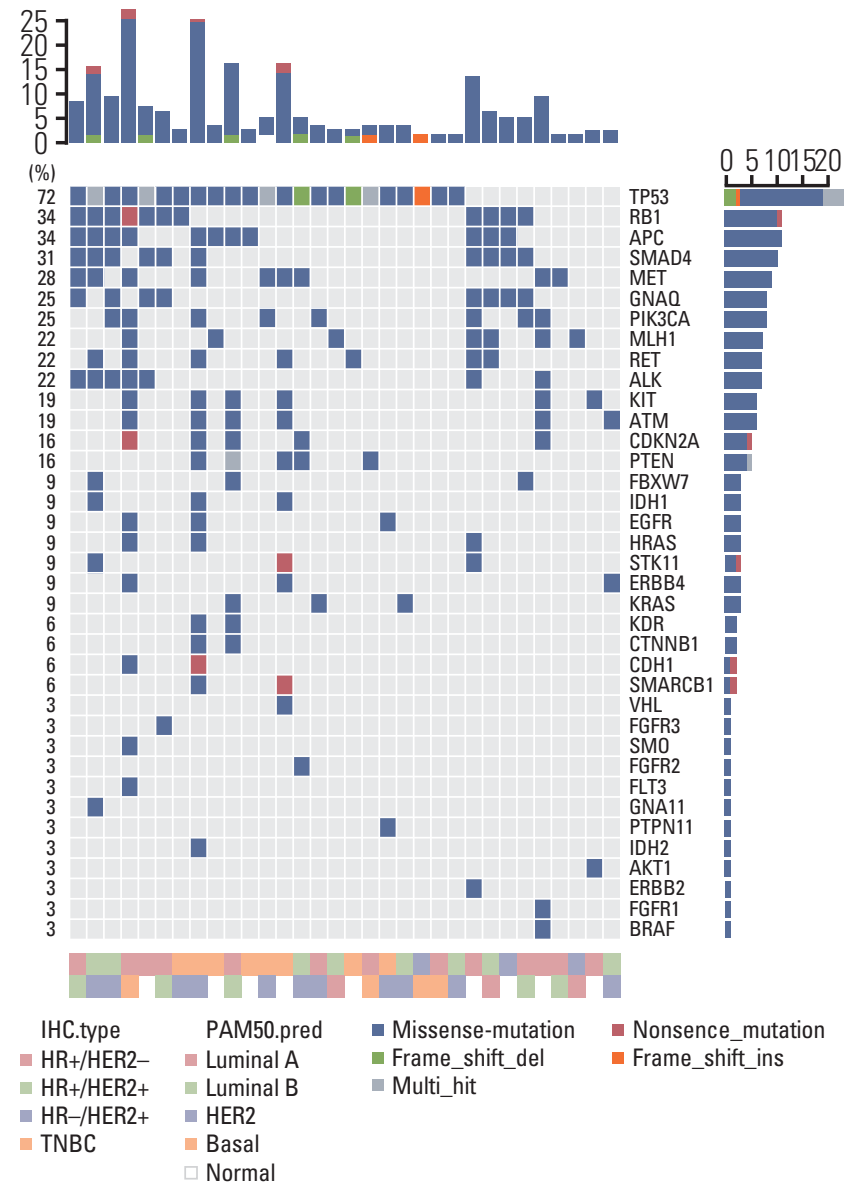

Fig. 2. (Continued from the previous page)

between concordant and discordant groups in all IHC-subtypes or HR+/HER2+ and HR-/HER2+ groups (Fig. 3B and C).

Fig. 4 shows the re-clustering of groups according to incidence of mutations. We defined eight groups as classified by IHC-based subtype and PAM50 concordance/discordance. According to hierarchical re-clustering with 44 mutations, concordant TNBC, concordant HR+/HER2+, and concordant HR-/HER2 + were grouped as a significantly similar entity cluster $(\mathrm{p}=0.03)$.

5. Discordant results between IHC-based subtype and PAM50 intrinsic subtype is associated with significantly shorter OS

To evaluate the impact of discordance on survival, we analyzed the OS according to discordance. The median duration of follow-up was 125 months (95\% confidence interval [CI], 121.4 to 129.9). The patients with discordant results had shorter OS than patients with concordant results, but the difference was not statistically significant (5-year OS rate, 83.6\% vs. $87.7 \%$; $\mathrm{p}=0.26)$ (Fig. 5A). Among HR+ patients ( $\mathrm{n}=343)$, the patients with basal-like subtype by PAM50 $(n=17)$ showed significantly shorter median OS compared to non-basal-like subtypes (5-year OS rate, 75.6\% vs. 92.2\%; $\mathrm{p}=0.01$ ) (Fig. 5B). In patients with luminal A or B by PAM50, concordance with IHC demonstrated a better OS (median OS, not reached vs. 73.6 months; $\mathrm{p}<0.001$ ) (Fig. 5C).

\section{Discussion}

Currently commercial assays for intrinsic molecular subtypes are available for patients with early HR+/HER2- and node-negative breast cancer, and adjuvant systemic therapy is recommended in accordance with the result $[18,19]$. However, only a small portion of breast cancer patients benefit from the multi-gene assay, and the majority of patients still rely on IHC-based subtypes in the clinic. In the current study, we found that discordance of IHC-based subtype with intrinsic subtype is considerable and is correlated with survival.

We found an overall discordance rate of $39 \%$ between IHC-based subtype and intrinsic molecular subtype. Our study showed that discordance could be a reason for the under-treatment or over-treatment of breast cancer. For example, 29\% of the patients with HER2-enriched subtype were HER2+ disease according to IHC (S5 Fig.). Patients with HER2-enriched / HER2-negative by IHC could experience under-treatment with hormone therapy or omission of antiHER2 antibody. Such an oversight would not guarantee adequate treatment for breast cancer. The 2011 St. Gallen guideline recommended the treatment of breast cancer according to pathologic determination of estrogen receptor (ER), progesterone receptor, HER2, and Ki67 [20]. Our results suggest that the guideline of St. Gallen should consider patients with discordance.

The discrepancy between IHC-based subtype and intrinsic molecular subtype has recently been analyzed in several studies. The study of Parker-PAM50 [7] were evaluated in accordance with IHC-based subtype, and the discordance rate of HR+ was $45.1 \%$, while that of HER2+ was $28.6 \%$ in analysis by Paquet and Hallet [21]. The HR+/HER2- subcohort demonstrated that $58.3 \%$ of Lumina A and $80.0 \%$ of HR/ HER2+ subcohorts were HER2-enriched type $[7,21]$. In recent studies of patients with HER2+ by IHC, about $51 \%$ had HER2-enriched intrinsic subtype [22]. In our study, 55\% of HR+ / HER2- patients were luminal A, and 74\% of HR/ HER2+ patients were HER2-enriched. These results are consistent with previous studies. 


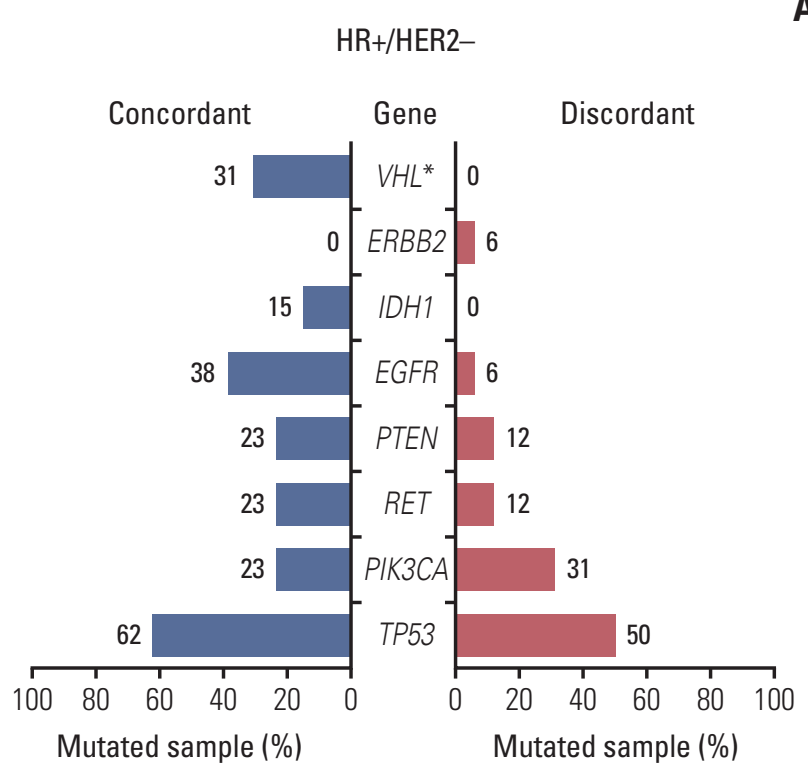

HR-/HER2+

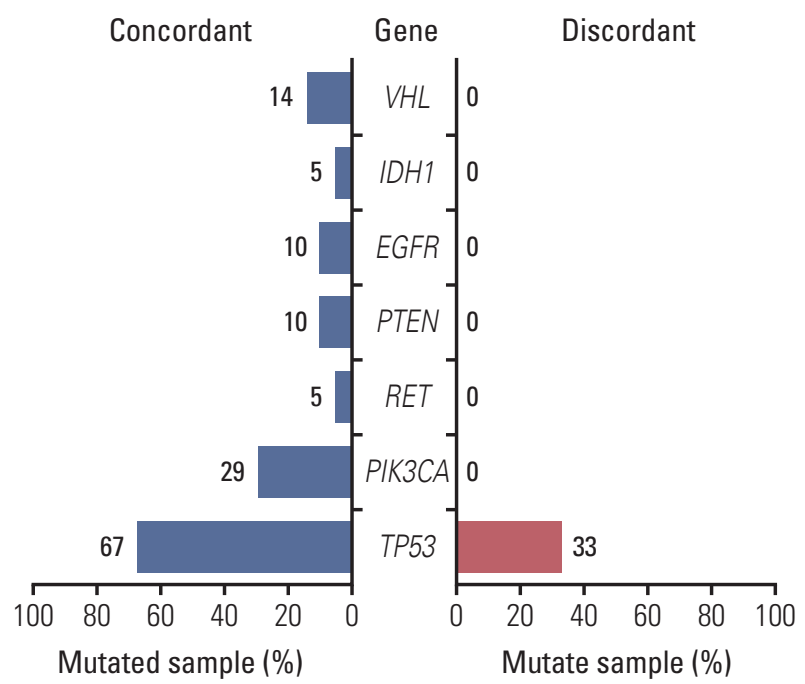

A

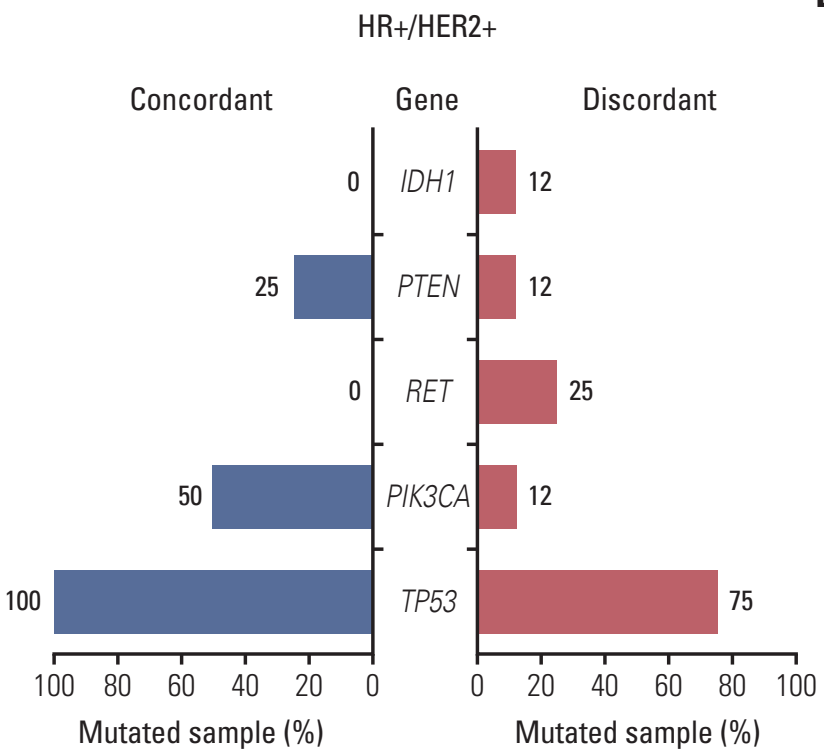

C

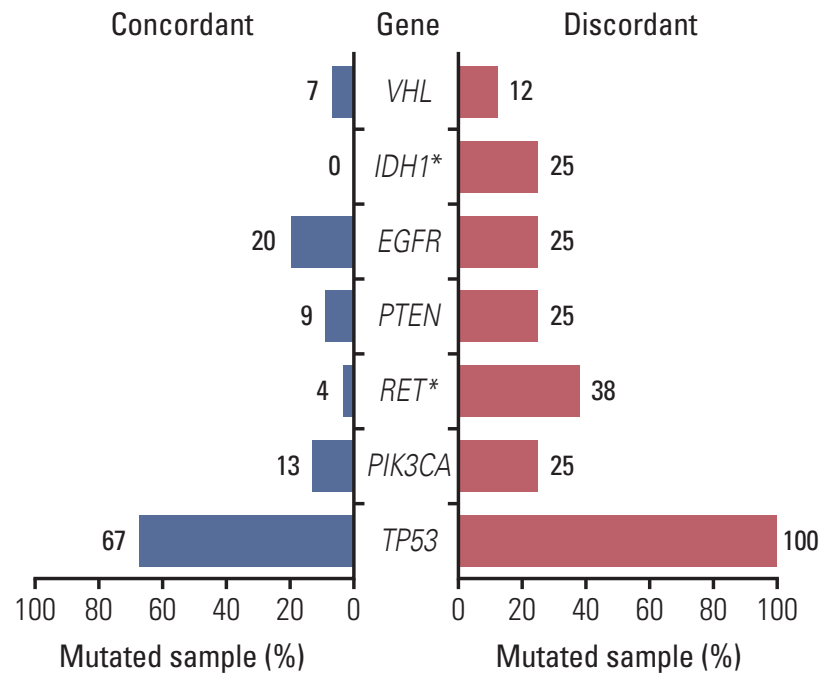

Fig. 3. The prevalence of mutated genes in concordant/discordant tumors of hormone receptor (HR)+/human epidermal growth factor receptor 2 (HER2)- (A), HR+/HER2+ (B), HR-/HER2+ (C), and TNBC (D). *p $<0.05$.

Marked heterogeneity was shown in mutational profile based on DNA sequencing in the current study. The genetic alterations that we found were mostly known driver mutations of breast cancer including TP53, PIK3CA, MLH1, and $R B 1[23,24]$. Mutations of the IDH1 and RET genes were most frequently observed in discordant TNBC patients. IDH1 mutations are commonly observed in brain tumors including glioblastoma multiforme and are known to be related with prognosis $[25,26]$. The IDH1 mutation in breast cancer has been reported, but the role of the mutation is unclear. RET oncogenic mutations are also well known genomic alterations in human cancer and multiple endocrine neoplasia type 2A, 2B [27,28]. Cabozantinib and vandetanib, oral tyrosine kinase inhibitors with multi-target including RET, are approved for the treatment RET-mutated metastatic medullary thyroid carcinoma by the U.S. FDA. RET mutations in 
A

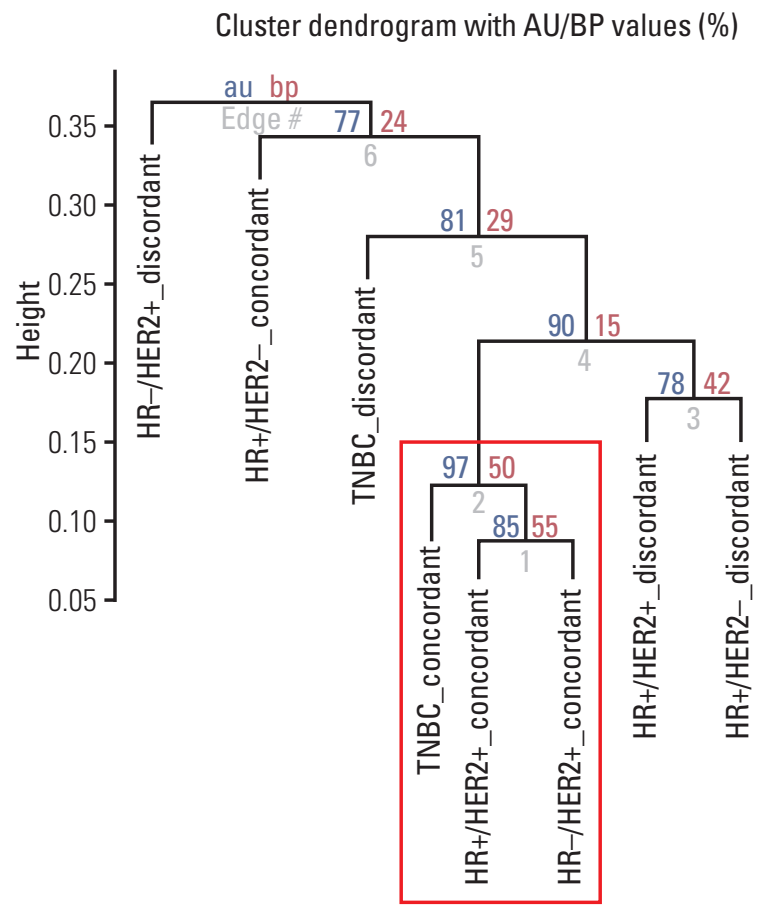

Distance: correlation Cluster method: average

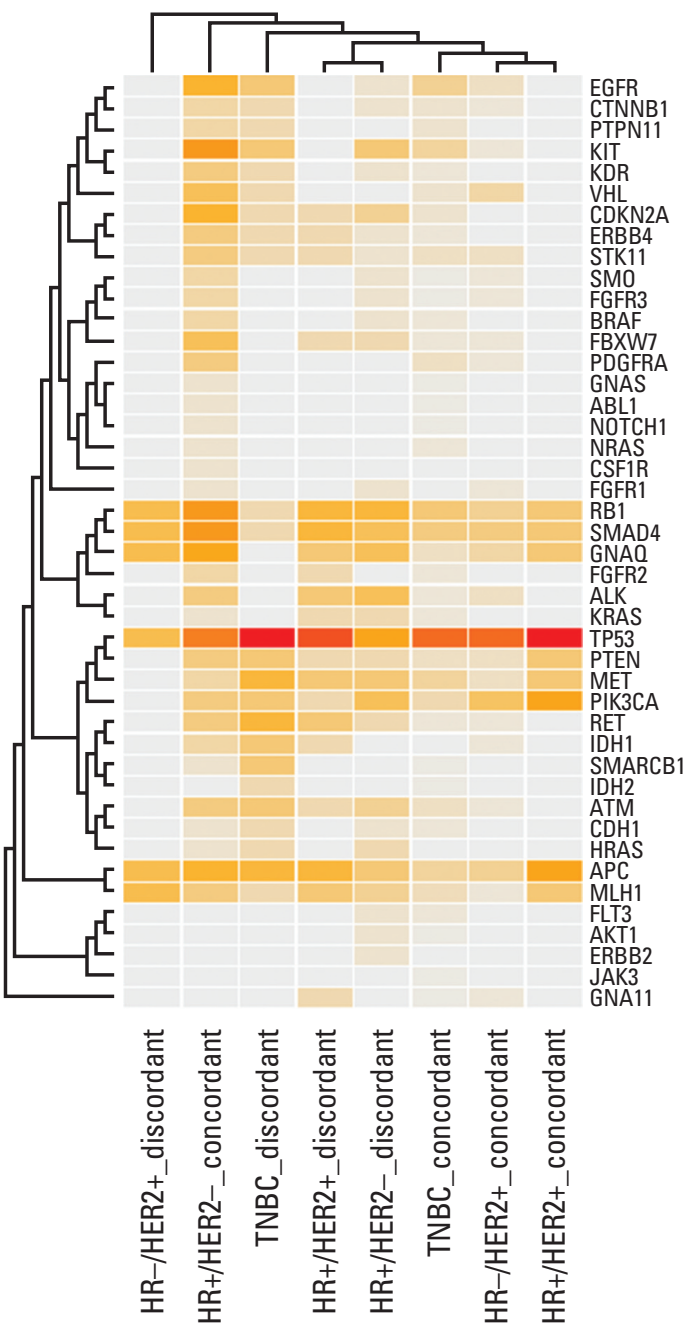

Fig. 4. Re-classification for hierarchical clustering of data based on the incidence of mutated genes in concordant and discordant tumors. (A) The dendrogram of discordant pattern based on mutated genes using 10,000 multi-scale bootstrap re-sampling; blue letter: approximately unbiased (AU) values, red letters: bootstrap probability (BP) values. Clusters with AU values greater than 0.95 are highlighted with a red rectangle and are strongly supported. (B) The heatmap of discordant pattern with mutation incidence.

breast cancer have been reported, and some studies have suggested that RET mutations are correlated with ER expression $[29,30]$. Three patients were discordant TNBC as luminal A intrinsic type in our study, but we could not check if they had the RET mutation.

In addition, we tried to regroup the patients based on the incidence of mutated genes in concordant and discordant tumors. The results showed significant clusters that consisted of concordant TNBC, concordant HR+ / HER2+, and concordant HR-/HER2+ ( $\mathrm{p}=0.003$ ) (Fig. 5A). The phylogenetic tree depicted a greater genetic distance between discordant HR/HER2+ patients and the other subgroups. Concordant
HR+/HER2- patients were genetically far from discordant HR+/HER2- patients, demonstrating the significance of discordance between IHC and intrinsic subtypes.

We evaluated the OS of patients according to discordance with a median follow-up 10 years, and the results showed that discordance could influence survival of breast cancer. Notably, we found that the discordant results had significantly worse OS in two populations. First discordant group (HR- by IHC but luminal A or B intrinsic subtype) showed 73.6 months of median OS ( $95 \%$ CI, 52.6 to 94.6 months) but concordant group (HR+ and luminal A or B) not reached median OS ( $<<0.001)$ (Fig. 5B). Second discordant group 
A
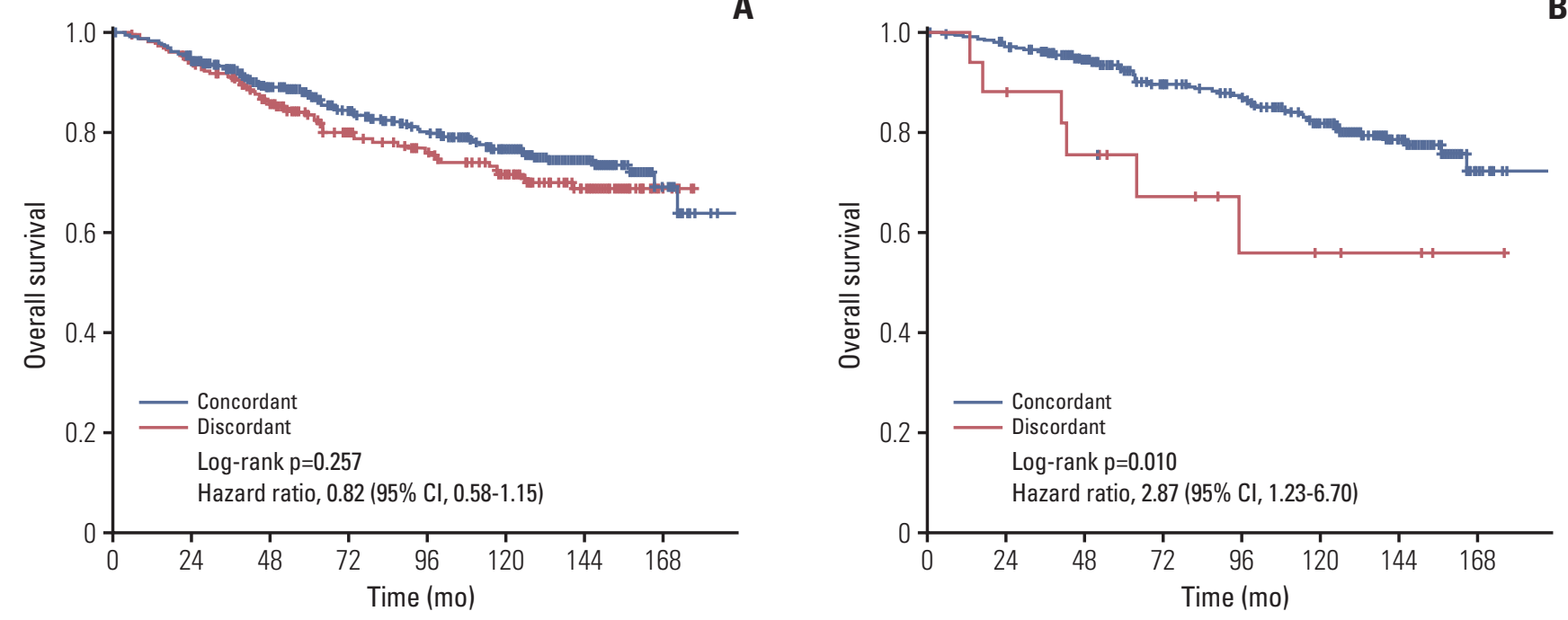

C

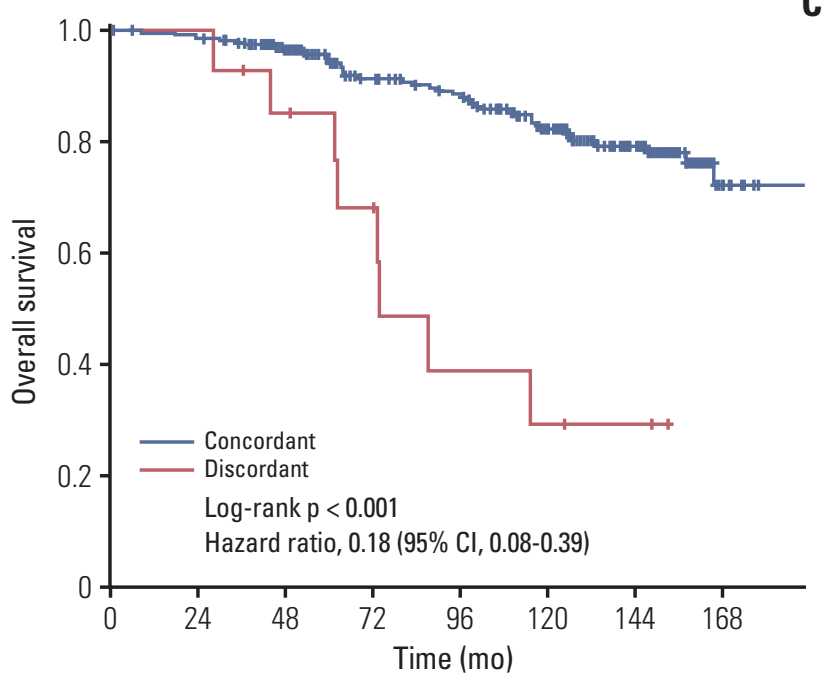

Fig. 5. Kaplan-Meier curve of overall survival (OS) according to discordance. (A) All patients ( $\mathrm{n}=607)$. (B) OS in basal-like and non-basal-like types in hormone receptor (HR)+ patients $(n=343)$. (C) OS in HR+ and HR-by immunohistochemistry in luminal A or B patients (n=283). CI, confidence interval.

(HR+ but basal-like intrinsic subtype) had significantly worse OS (5-year OS rate, 92.2\% vs. 75.6\%; $\mathrm{p}=0.010$ ) (Fig. 5C). The potential explanation for this result is under-treatment of patients with discordant results and better survival of HR+ patients with endocrine therapy. Similarly, the 5-year OS rate was $87.2 \%$ in concordant HER2-enriched patients (IHC was HER2+ and PAM50 was HER2-enriched), while the patients with discordant result (IHC was not HER2+ but PAM50 was HER2-enriched) had a 75.4\% 5-year OS rate $(\mathrm{p}=0.807)$ (S6 Fig.). This result could be attributed to whether anti-
HER2 treatment was given. Because HER2-enriched patients with a discordant result might not receive anti-HER2 treatment, they showed lower survival at 5 years.

Our study is retrospective and the tumor tissues were acquired from six clinical cohorts rather than from a single cohort, so interpretation of the results should be judicious. Although we tried to reduce the batch effect and to exclude normal samples for insurance of comparability between expression levels of genes across patients, the difference of sample quality among six cohorts could be a limitation. The 
drawback of the method of Parker et al. [7] was that PAM50 prediction might be not robust according to the cohort configuration, particularly in cohorts with specific IHC alone. In order to overcome the problems of the Parker's method and the disadvantage of not performing the commercial PAM50 test, we applied the TCGA adjustment to the PAM50 prediction. Most Samsung Medical Center study cohorts showed similar concordance regardless of TCGA adjustment, and we could get better concordance in patients with only specific IHCs by applying TCGA adjustment (S7 Fig.). However, the predictability of PAM50 could be decreased in a specific subcohort (e.g., a specific subtype) [21], our multi-cohort might overcome the shortcoming of PAM50 prediction. The small sample size for mutation profile analysis (a total of 118 samples) unlike PAM50 prediction ( $\mathrm{n}=607)$ was also a limitation of our study. Hence, caution is necessary when drawing a conclusion about correlation between discordance and mutation pattern.

In conclusion, we analyzed the distribution of intrinsic molecular subtypes within IHC-based subtype and survival outcomes in accordance with discordance. We also found several gene alterations related to discordance through analysis of mutational profiling, and the result demonstrated the patients with the same IHC subtype had genetically different features. To the best of our knowledge, this is the first study to investigate analyze the impact of discordance between IHC-based subtypes and PAM50 prediction. Our result suggests that discordance between intrinsic subtype and IHCbased subtype could result in inadequate treatment and poor survival of breast cancer, so the treatment guidelines might be need to be revised to ensure proper intrinsic subtype characterization.

\section{Electronic Supplementary Material}

Supplementary materials are available at Cancer Research and Treatment website (https://www.e-crt.org).

\section{Conflicts of Interest}

Conflict of interest relevant to this article was not reported.

\section{Acknowledgments}

This research was funded by Samsung Biomedical Research Institute.

\section{Author Details}

${ }^{1}$ Division of Hematology-Oncology, Department of Internal Medicine, Samsung Medical Center, Sungkyunkwan University School of Medicine, Seoul, '2Department of Internal Medicine, Chungbuk National University Hospital, Chungbuk National University College of Medicine, Cheongju, ${ }^{3}$ Samsung Genome Institute, Samsung Medical Center, Seoul, ${ }^{4}$ Division of Breast Surgery, Department of Surgery, Samsung Medical Center, Sungkyunkwan University School of Medicine, Seoul, Korea

\section{References}

1. Torre LA, Bray F, Siegel RL, Ferlay J, Lortet-Tieulent J, Jemal A. Global cancer statistics, 2012. CA Cancer J Clin. 2015;65:87-108.

2. Cancer Genome Atlas Network. Comprehensive molecular portraits of human breast tumours. Nature. 2012;490:61-70.

3. Perou CM, Sorlie T, Eisen MB, van de Rijn M, Jeffrey SS, Rees $\mathrm{CA}$, et al. Molecular portraits of human breast tumours. Nature. 2000;406:747-52.

4. Prat A, Parker JS, Fan C, Perou CM. PAM50 assay and the three-gene model for identifying the major and clinically relevant molecular subtypes of breast cancer. Breast Cancer Res Treat. 2012;135:301-6.

5. Sotiriou C, Pusztai L. Gene-expression signatures in breast cancer. N Engl J Med. 2009;360:790-800.

6. Paik S, Shak S, Tang G, Kim C, Baker J, Cronin M, et al. A multigene assay to predict recurrence of tamoxifen-treated, node-negative breast cancer. N Engl J Med. 2004;351:2817-26.

7. Parker JS, Mullins M, Cheang MC, Leung S, Voduc D, Vickery $\mathrm{T}$, et al. Supervised risk predictor of breast cancer based on intrinsic subtypes. J Clin Oncol. 2009;27:1160-7.

8. Prat A, Perou CM. Deconstructing the molecular portraits of breast cancer. Mol Oncol. 2011;5:5-23.

9. Gnant M, Filipits M, Greil R, Stoeger H, Rudas M, Bago-Horvath $\mathrm{Z}$, et al. Predicting distant recurrence in receptor-positive breast cancer patients with limited clinicopathological risk: using the PAM50 Risk of Recurrence score in 1478 postmenopausal patients of the ABCSG-8 trial treated with adjuvant endocrine therapy alone. Ann Oncol. 2014;25:339-45.

10. Dowsett M, Sestak I, Lopez-Knowles E, Sidhu K, Dunbier AK, Cowens JW, et al. Comparison of PAM50 risk of recurrence score with oncotype DX and IHC4 for predicting risk of distant recurrence after endocrine therapy. J Clin Oncol. 2013;31:2783-90.

11. Nielsen TO, Parker JS, Leung S, Voduc D, Ebbert M, Vickery $\mathrm{T}$, et al. A comparison of PAM50 intrinsic subtyping with immunohistochemistry and clinical prognostic factors in tamoxifen-treated estrogen receptor-positive breast cancer. Clin Cancer Res. 2010;16:5222-32. 
12. Leong SP, Shen ZZ, Liu TJ, Agarwal G, Tajima T, Paik NS, et al. Is breast cancer the same disease in Asian and Western countries? World J Surg. 2010;34:2308-24.

13. Min SY, Kim Z, Hur MH, Yoon CS, Park EH, Jung KW, et al. The basic facts of Korean breast cancer in 2013: results of a nationwide survey and breast cancer registry database. J Breast Cancer. 2016;19:1-7.

14. Park YH, Lee SJ, Jung HA, Kim SM, Kim MJ, Kil WH, et al. Prevalence and clinical outcomes of young breast cancer (YBC) patients according to intrinsic breast cancer subtypes: Single institutional experience in Korea. Breast. 2015;24:213-7.

15. Harris LN, Ismaila N, McShane LM, Andre F, Collyar DE, Gonzalez-Angulo AM, et al. Use of biomarkers to guide decisions on adjuvant systemic therapy for women with earlystage invasive breast cancer: American Society of Clinical Oncology clinical practice guideline. J Clin Oncol. 2016;34: 1134-50.

16. Sohn I, Kim J, Jung SH, Park C. Gradient lasso for Cox proportional hazards model. Bioinformatics. 2009;25:1775-81.

17. Johnson WE, Li C, Rabinovic A. Adjusting batch effects in microarray expression data using empirical Bayes methods. Biostatistics. 2007;8:118-27.

18. Goldhirsch A, Winer EP, Coates AS, Gelber RD, Piccart-Gebhart M, Thurlimann B, et al. Personalizing the treatment of women with early breast cancer: highlights of the St Gallen International Expert Consensus on the Primary Therapy of Early Breast Cancer 2013. Ann Oncol. 2013;24:2206-23.

19. Krop I, Ismaila N, Andre F, Bast RC, Barlow W, Collyar DE, et al. Use of biomarkers to guide decisions on adjuvant systemic therapy for women with early-stage invasive breast cancer: American Society of Clinical Oncology clinical practice guideline focused update. J Clin Oncol. 2017;35:2838-47.

20. Goldhirsch A, Wood WC, Coates AS, Gelber RD, Thurlimann B, Senn HJ, et al. Strategies for subtypes: dealing with the diversity of breast cancer: highlights of the St. Gallen International Expert Consensus on the Primary Therapy of Early Breast Cancer 2011. Ann Oncol. 2011;22:1736-47.
21. Paquet ER, Hallett MT. Absolute assignment of breast cancer intrinsic molecular subtype. J Natl Cancer Inst. 2015;107:357.

22. Carey LA, Berry DA, Cirrincione CT, Barry WT, Pitcher BN, Harris LN, et al. Molecular heterogeneity and response to neoadjuvant human epidermal growth factor receptor 2 targeting in CALGB 40601, a randomized phase III trial of paclitaxel plus trastuzumab with or without lapatinib. J Clin Oncol. 2016;34:542-9.

23. Ramaswamy S, Ross KN, Lander ES, Golub TR. A molecular signature of metastasis in primary solid tumors. Nat Genet. 2003;33:49-54.

24. van 't Veer LJ, Dai H, van de Vijver MJ, He YD, Hart AA, Mao $\mathrm{M}$, et al. Gene expression profiling predicts clinical outcome of breast cancer. Nature. 2002;415:530-6.

25. Parsons DW, Jones S, Zhang X, Lin JC, Leary RJ, Angenendt $\mathrm{P}$, et al. An integrated genomic analysis of human glioblastoma multiforme. Science. 2008;321:1807-12.

26. Combs SE, Rieken S, Wick W, Abdollahi A, von Deimling A, Debus J, et al. Prognostic significance of IDH-1 and MGMT in patients with glioblastoma: one step forward, and one step back? Radiat Oncol. 2011;6:115.

27. Kan Z, Jaiswal BS, Stinson J, Janakiraman V, Bhatt D, Stern $\mathrm{HM}$, et al. Diverse somatic mutation patterns and pathway alterations in human cancers. Nature. 2010;466:869-73.

28. Plaza-Menacho I, Burzynski GM, de Groot JW, Eggen BJ, Hofstra RM. Current concepts in RET-related genetics, signaling and therapeutics. Trends Genet. 2006;22:627-36.

29. Esseghir S, Todd SK, Hunt T, Poulsom R, Plaza-Menacho I, Reis-Filho JS, et al. A role for glial cell derived neurotrophic factor induced expression by inflammatory cytokines and RET/GFR alpha 1 receptor up-regulation in breast cancer. Cancer Res. 2007;67:11732-41.

30. Tozlu S, Girault I, Vacher S, Vendrell J, Andrieu C, Spyratos $\mathrm{F}$, et al. Identification of novel genes that co-cluster with estrogen receptor alpha in breast tumor biopsy specimens, using a large-scale real-time reverse transcription-PCR approach. Endocr Relat Cancer. 2006;13:1109-20. 